\title{
Hibernating Myocardium and Role of 18F FDG PET Cardiac Imaging - A review
}

\author{
Raihan Hussain
}

National Institute of Nuclear Medicine and Allied Sciences, BAEC

Correspondence Address: Dr. Dr. Raihan Hussain, MBBS, DNM, FANMB, PhD., Professor \& Chief Medical Officer, Head, Nuclear Cardiology Division and PET-CT Divisions, National Institute of Nuclear Medicine and Allied Sciences, BSM Medical University

Campus, Dhaka, Bangladesh

President, Society of Nuclear Medicine, Bangladesh, Vice President, Bangladesh Society of Ultrasonography Secretary General, Asia and Oceania Federation of Nuclear Medicine and Biology (AOFNMB) Treasurer, Asian Regional Cooperative Council for Nuclear Medicine (ARCCNM) Member, Governing Body, World Association of Radiopharmaceutical and Molecular Therapy (WARMTH), Tel:+ 88028621697 (off); + 8801711535101 (cell); e-mail: raihan_h@yahoo.com

\begin{abstract}
The term "Hibernation" is a challenging term but not infrequently used in Nuclear Cardiology. It often is used synonymously for tissue viability, although technically there are some differences. Viability is a prospective definition, but it does not imply evidence of functional recovery after interventions whereas hibernation is a retrospective definition based on evidence of functional recovery after interventions. But for practical clinical purposes both carries the similar meaning. The aim of this review article is to summarize our current understanding of the concept of hibernation and its clinical implications in patients with coronary artery disease (CAD) and the role of FDG PET study in its proper evaluation.
\end{abstract}

\section{INTRODUCTION}

Myocardial ischemia is a clinical syndrome manifesting a variety of tissue effects and global cardiac effects that impair cardiac function. When ischemia is severe and prolonged, it causes myocyte death and results in loss of contractile function and tissue infarction. In cases of less severe ischemia, some myocytes remain viable but have depressed contractile function. Heyndrickx et al described a phenomenon of prolonged depression of regional function after a reversible episode of ischemia in dogs (1); later, this was called myocardial stunning $(2,3,4,5)$. Repetitive stunning may evolve into hibernating myocardium.

Hibernating Myocardium typically represents a chronic process whereas stunned myocardium is typically related to an acute infarction or ischaemic insult. Differentiation from stunned myocardium can be difficult, but in which usually there is maintained, or nearly maintained, perfusion with a wall motion abnormality (hibernating myocardium will have abnormal perfusion and a wall motion abnormality). On stress and rest, a perfusion defect will be present (e.g. on both stress-rest images using $99 \mathrm{mTc}$ sestamibi or $99 \mathrm{~m} \mathrm{Tc}$ tetrofosmin SPECT or $82 \mathrm{Rb}$ or $13 \mathrm{~N}$ ammonia positron emission tomographycomputed tomography (PET-CT). However, due to retained viability, the tissue will demonstrate uptake of FDG (Table 1), which will discriminate between infarction and hibernating myocardium (6).

Hibernating and stunned myocardium are potential sequele of ischemia. In simple words these can be expressed as transient ischemia (stunned myocardium) with restored blood flow with transient contractile dysfunction, chronic ischemia with contractile dysfunction (hibernating myocardium) and infarction (cell death). Clinically, discerning the etiology of depressed myocardial contractile function is difficult, whether it is due to stunned myocardium, silent ischemia, or hibernating myocardium. Finding all three entities in the same patient with chronic myocardial dysfunction is not uncommon. The critical difference is that blood flow is normal or near normal with stunning but is reduced in the other two conditions. Some authors 
have suggested that stunning, silent ischemia, and hibernating myocardium are processes by which the myocardium adapts to reduced myocardial oxygen supply to maintain cellular integrity. However, this theory has not yet been confirmed by animal and human experiments (6).

Acute myocardial ischemia rapidly impairs contractile function (7). This dysfunction can persist for several hours after transient nonlethal ischemia but eventually is followed by full functional recovery (1). The latter phenomenon is known as myocardial stunning $(8,9$, 10). In patients with $\mathrm{CAD}$, repeated episodes of demand ischemia may lead to cumulative stunning that could be a substrate in the development of chronic postischemic LV dysfunction (11).

\section{DISCUSSION}

\section{Mechanisms for myocardial stunning and hibernation}

There are two major hypotheses for myocardial stunning:

(i) a oxygen-free radical hypothesis and (ii) a calcium overload hypothesis $(12,13,14,15)$. Post ischemic dysfunction may be due to cytotoxic oxygen-derived free radicals (ie, hydroxyl radicals, superoxide anions) that are thought to be generated during occlusion or, upon reperfusion. Such radicals cause lipid per oxidation, altering their function and structure.

Normal cardiac contraction depends on the maintenance of calcium cycling and homeostasis across the mitochondrial membrane and sarcoplasmic reticulum during each cardiac cycle. Brief ischemia followed by reperfusion damages $\mathrm{Ca} 2+$ pump and ion channels of the sarcoplasmic reticulum. This results in the electromechanical uncoupling of energy generation from contraction that characterizes myocardial stunning. Calcium accumulates in the cell at the time of reperfusion and that is followed by a partial failure of normal beat-to-beat calcium cycling, which perhaps occurs at the level of the sarcoplasmic reticulum. This mechanism is proposed to account for contractile dysfunction. Structurally, myocytes in stunned myocardium appear normal when examined by light microscopy. The appearance of glycogen vacuoles adjacent to mitochondria and of myofibrillary loss are noted in most cases of hibernating myocardium when examined by electron microscopy. Several controversies exist regarding these histologic changes $(16,17)$.

Several animal models have been proposed to demonstrate the physiologic significance of coronary stenosis, in which a regulation of flow and downregulation of metabolism lead to hibernating myocardium. The key determinant of this adaptive process is a reduction in the coronary perfusion reserve that results from critical coronary stenosis. Conti demonstrated that the production of an acute and critical reduction in coronary flow reserve (CFR) in chronically instrumented pigs leads to an accelerated progression of chronic stunning proceeding to hibernation in less than 2 weeks $(18,19,20,21,22,23)$. The time frame for the transition from stunning to hibernation can be fairly short, and it is directly related to the degree of flow impairment in a stenotic coronary artery that supplies the dysfunctional segment. As the ischemic threshold decreases with a reduction in the CFR, repetitive stunning results in a delay in the recovery of function that becomes longer than the interval between ischemic episodes $(12,13,14)$.

In patients with severe $\mathrm{CAD}$, therefore, the limited flow reserve leads to development of myocardial ischemia, even with a small increase in the oxygen demand, such as in ordinary daily activities and exercise. Thus, intermittent episodes of ischemia and, consequently, post ischemic stunning (which should occur frequently in patients with severe CAD) might play a role in the development of chronic reversible LV dysfunction. Collectively, these findings indicate that chronic repetitive ischemia that progresses to hibernating myocardium is associated with regional down-regulation of the sarcoplasmic reticulum, with changes in calcium regulation and gene expression. These changes are accompanied by modest increases in myocyte apoptosis and a reduction in the regional myocyte nuclear density. These same structural and functional findings occur in patients with dilated cardiomyopathy of ischemic origin (6). 


\section{Features of Hibernation}

Hibernating myocardium is characterized by the following:

- Episodic and/or chronically reduced blood flow, which is directly responsible for the decrease in the myocardial contractile function.

- Tissue ischemia and resultant remodeling without necrosis, which causes prioritization of metabolic process in the myocardial cell relative to contractile function.

- Residual contractile reserve in response to inotropic stimulation (in at least half of clinical cases).

- Recovery of contractile function after successful revascularization.

Hibernating myocardium is suspected in all patients with CADs and moderate-to-severe chronic LV dysfunction. As many as $50 \%$ of patients with previous infarction may have areas of hibernating tissue mixed with scar tissue, even in the presence of $\mathrm{Q}$ waves on the electrocardiogram. The extent and severity of ventricular dysfunction can vary considerably. Hibernating myocardium may be limited to a discrete portion of ventricle with normal systolic function, or it may involve global impairment of LV function and result in the clinical syndrome of heart failure. Thus, the clinical dilemma exists regarding attempted coronary artery bypass grafting $(\mathrm{CABG})$ in high-risk patients to restore global ventricular function versus orthotopic cardiac transplantation $(24,25,26,27)$.

\section{Viability Studies}

Myocardial perfusion imaging (MPI) is used to estimate myocardial blood flow (MBF) and metabolic activity. Several factors limit the use of thallium and technetiumbased agents to accurately quantify MBF. Positron emission tomography (PET) overcomes important limitations by providing attenuation correction, enabling quantification of the concentration of the radiotracer in the organ of interest. Initial PET studies revealed that hibernating segments correspond to areas with qualitatively reduced perfusion, in the presence of preserved fluorodeoxyglucose (FDG) uptake. This resulted in the concept of perfusion and metabolism mismatch in which flow is reduced but metabolic integrity is retained. In addition to cardiac PET, there are other diagnostic imaging techniques that are also commonly used to determine myocardial viability such as SPECT, cardiac magnetic resonance imaging (MRI) and echocardiography. The diagnostic accuracy of 18F FDG PET compares well with other established techniques for viability assessment (SPECT, echocardiography, and cardiac MRI), with sensitivity being superior to other techniques, although cardiac PET is not as widely available as other techniques (28).

Compared with thallium imaging, FDG-PET imaging provides better results for the differentiation of viable myocardium from scarred myocardium. Brunken et al published data from a comparison of tomographic thallium images with PET images; $47 \%$ of the irreversible thallium defects were identified as viable on PET images (29). Tamaki et al subsequently confirmed these findings in 2 comparative studies of SPECT and PET in which $38-42 \%$ of the irreversible thallium defects had enhanced FDG uptake suggestive of viable myocardium $(30,31)$. Thus, conventional stress-redistribution $201 \mathrm{Tl}$ imaging has a low predictive value in the identification of viable myocardium when compared with PET.

Invasive coronary angiography is the gold standard for the identification of obstructive coronary lesions, however, it lacks the sensitivity and specificity for the early detection of coronary artery disease, quantitative analysis of plaque composition and cardiac function assessment $(32,33)$. The most recently published PROSPECT (Providing Regional Observations to Study Predictors of Events in the Coronary Tree) trial highlights the value of non- or less-invasive imaging modalities to evaluate high-risk plaque for more accurate risk stratification of patients with coronary artery disease, as invasive angiography was found to have poor predictive accuracy in identifying patients with high risk of developing acute cardiac events (29). 
In routine clinical practice, cardiac SPECT or PET is commonly used for the assessment of myocardial perfusion and metabolism, while invasive coronary angiography is reserved for confirming disease extent and performing revascularization.

\section{F FDG PET}

Tillisch, et al. (34) in their first pioneering study reported the value of $18 \mathrm{~F}$ FDG PET to predict reversibility of cardiac wall motion abnormalities in 17 patients with ischemic cardiomyopathy. The overall sensitivity and specificity of $18 \mathrm{~F}$ FDG PET to demonstrate functional recovery after surgery was $95 \%$ and $80 \%$, respectively. A meta-analysis of 24 studies has reported a weighted sensitivity and specificity of $92 \%$ and $63 \%$, respectively, with a positive and negative predictive value of $74 \%$ and $87 \%$, respectively, for the diagnosis of hibernating myocardium and prediction of patient outcomes (35).

Early studies demonstrated that myocardial ischemia and infarction could be distinguished by analysis of positron emission tomography (PET) images of the perfusion tracer $13 \mathrm{~N}$-labeled ammonia (13NH3) and the glucose analog 18Ffluorodeoxyglucose (FDG) acquired after an oral glucose load (34). Regions that showed a concordant reduction in both myocardial blood flow and FDG uptake ("flowmetabolism match") were considered to be irreversibly injured, whereas regions in which FDG uptake was relatively preserved or increased despite having a perfusion defect ("flow-metabolism mismatch") were considered ischemic but still viable (36). Three patterns of myocardial viability may occur with a PET perfusion-metabolism mismatch protocol. The perfusion metabolism match pattern demonstrates either a concordantly reduced, absent, or regional myocardial perfusion and FDG uptake. If severe, this implies transmural infarction and irreversible LV function. A pattern in which mild-to-moderate flow is matched to metabolism suggests the presence of both viable and nonviable tissue in a given region of myocardium. On the other hand, when regional myocardial FDG uptake is disproportionately enhanced compared with blood flow, the pattern is termed perfusionmetabolism mismatch. This pattern closely resembles that of hibernating myocardium.
Table 1: Summary of MPI and FDG Metabolism interpretations.

\begin{tabular}{|l|l|l|l|l|}
\hline Condition & Perfusion Stress & Perfusion Rest & Wall Motion & Metabolism \\
\hline Normal & Y & Y & Y & Y \\
\hline Ischemia & N & Y & Y & Y \\
\hline Stunning & N & Y & N & Y \\
\hline Hibernating & N & N & N & Y \\
\hline Infarction & N & N & N & N \\
\hline
\end{tabular}

\section{Protocol}

The patients are fasted for at least $6 \mathrm{~h}$ before the scan and baseline blood sugar is checked. About 45-60 min after injection of the 50-75 $\mathrm{g}$ of glucose loading, blood sugar is checked. If it is $<140 \mathrm{mg} / \mathrm{dL}, 400 \mathrm{MBq}(10 \mathrm{mCi})$ of $18 \mathrm{~F}$ FDG is injected intravenously. If it is $>140 \mathrm{mg} / \mathrm{dL}$, regular insulin is injected intravenously according to blood glucose level $(2,3$ and $5 \mathrm{U}$ of regular insulin for 140-160, 160-180, and 180-200 mg/dL of blood glucose, respectively). About 45-60 min after 18F FDG injection myocardial 18F FDG PET study is performed. PET acquisition parameters are as follows: myocardium is covered in one bed position ( 5 min per bed position) with ECG gating (8 frames/RR cycle).

The American College of Cardiology/American Heart Association Task Force on Practice Guidelines (ACC/AHA/ASNC) recommends a semi-quantitative analysis of PET studies based on a validated segmental scoring system. A 17-segment model analysis was proposed using a 5-point scale system in direct proportion to the observed count density of the segment: $0=$ normal perfusion, $1=$ mild defect, $2=$ moderate defect, $3=$ severe defect and $4=$ absent uptake (37).

Study findings clearly indicate that oxidative metabolism is preserved in ischemic and hibernating myocardium. In ischemic and hibernating myocardium, regional substrate utilization shifts from free fatty acids to glucose $(38,39$, 40). The production of the glucose protein transporter (GLUT) protein is increased, as demonstrated by Schwaiger et al, as is the expression of myocardial glucose transporter messenger RNA protein in patients with advanced CAD (41). Glucose utilization 
is, in turn, influenced by a number of factors, such as coronary perfusion, cardiac work, and insulin and hormonal effects. FDG is a glucose analogue that is intracellularly phosphorylated to FDG-6-phosphate in the myocardium. An increased uptake of 18F FDG in relation to perfusion or flow-metabolism mismatch is indicative of hibernating myocardium, whereas matched defects are indicative of scar tissue; values in between these represent healthy myocardial tissue mixed with fibrosis (42). Preoperative measurement of the regional flow and uptake of $18 \mathrm{~F}$ FDG can be used to accurately predict functional recovery after revascularization in patients with depressed ventricular function.

The uptake of FDG by the myocardium and scan quality depend on many factors such as dietary state, cardiac workload, sympathetic drive, and the presence and severity of ischemia (43). Many patients with heart failure are insulin resistant, and the amount of endogenous insulin released after oral glucose loading will not induce maximal stimulation of myocardial glucose/FDG uptake (44). To minimize these problems and to avoid the need of a simultaneous $13 \mathrm{NH} 3$ scan, which requires a cyclotron on site, an alternative protocol, the main feature of which is the use of the hyperinsulinemic euglycemic clamp, has been developed (45). During the clamp, plasma free fatty acid levels are dramatically reduced, and metabolism in insulin-sensitive tissues switches to glucose use (46, 47). FDG is injected during the steady-state phase of the clamp, resulting in high and rapid myocardial FDG uptake and low tracer concentration in the blood pool, leading to improved image quality even in patients with insulin resistance. The scan acquisition time is reduced to 15 to 20 minutes, starting 20 to 30 minutes after FDG injection. This approach has been tested in a European multicenter study (48).

The main strengths of PET compared with SPECT are its superior spatial resolution and attenuation correction. PET is generally regarded as the gold standard for viability assessment. Reduced availability of PET scanners and the need for a cyclotron on site have been the main limitations to wider clinical applications. Recently, however, the number of scanners available has increased to $\approx 1200$ in the United States and 400 in Europe. In the United States, the annual procedure volume for cardiology was estimated to be 154000 , of which $\approx 68000$ were viability and $\approx 86000$ were rubidium-82 perfusion scans (information from scanner manufacturers) (49).

Although 18F FDG PET is regarded as the gold standard for the diagnosis of myocardial viability (50), cardiac PET is not widely available as opposed to SPECT. Furthermore, the high cost of PET camera and cyclotron technology limit the extensive use of this technique in many clinical centers.

\section{CONCLUSION}

Cardiac PET utilizing 18F FDG is considered the most sensitive modality for detecting hibernating viable myocardium and predicting left ventricular functional recovery post-coronary revascularization.

\section{REFERENCES}

1. Heyndrickx GR, Millard RW, McRitchie RJ, Maroko PR, Vatner SF. Regional myocardial functional and electrophysiological alterations after brief coronary artery occlusion in conscious dogs. J Clin Invest 1975;56(4):978-85.

2. Laky D, Parascan L, Cândea V. Myocardial stunning. Morphological studies in acute experimental ischemia and intraoperatory myocardial biopsies. Rom J Morphol Embryol 2008; 49(2):153-8.

3. Camici PG, Prasad SK, Rimoldi OE. Stunning, hibernation, and assessment of myocardial viability. Circulation 2008;117(1):103-14.

4. Sandroni C, Sanna T, Cavallaro F, Caricato A. Myocardial stunning after successful defibrillation. Resuscitation 2008;76(1):3-4.

5. Lucats L, Ghaleh B, Monnet X, Colin P, Bizé A, Berdeaux A. Conversion of post-systolic wall thickening into ejectional thickening by selective heart rate reduction during myocardial stunning. Eur Heart J 2007;28(7):872-9.

6. Bhola R. (Apr 08, 2015). Hibernating and Stunned Myocardium Imaging. Website: emedicne.medscape.com

7. Tennant R, Wiggers CJ. Effect of coronary occlusion on myocardial contraction. Am J Physiol 1935;112: 351-361.

8. Braunwald E, Kloner RA. The stunned myocardium: prolonged, postischemic ventricular dysfunction. Circulation 1982; 66: 11461149.

9. Ambrosio G, Betocchi S, Pace L, Losi MA, Perrone-Filardi P, Soricelli A, Piscione F, Taube J, Squame F, Salvatore M, Weiss JL, Chiariello M. Prolonged impairment of regional contractile function after resolution of exercise-induced angina: evidence of myocardial stunning in patients with coronary arterydisease. Circulation 1996; 94: $2455-2464$

10. Barnes E, Hall RJ, Dutka DP, Camici PG. Absolute blood flow and oxygen consumption in stunned myocardium in patients with coronary artery disease. J Am Coll Cardiol 2002; 39: 420-427.

11. Barnes E, Dutka DP, Khan M, Camici PG, Hall RJ. Effect of repeated episodes of reversible myocardial ischemia on myocardial 
blood flow and function in humans. Am J Physiol Heart Circ Physiol 2002; 282: H1603-H1608.

12. Bolli R. Myocardial 'stunning' in man. Circulation 1992; 86(6):1671-91.

13. Bolli R. Mechanism of myocardial "stunning". Circulation 1990;82(3):723-38.

14. Bolli R. Oxygen-derived free radicals and myocardial reperfusion injury: an overview. Cardiovasc Drugs Ther 1991;2:249-68.

15. Kloner RA, Przyklenk K. Stunned and hibernating myocardium. Annu Rev Med 1991;42:1-8.

16. Chronic ischemic viable myocardium in man: aspect of dedifferentiation. Cardiovasc Pathol 1995;4:29-37.

17. Vanoverschelde JL, Wijns W, Borgers M, Heyndrickx G, Depré C, Flameng W. Chronic myocardial hibernation in humans. From bedside to bench. Circulation 1997; 95(7):1961-71.

18. Conti CR. The stunned and hibernating myocardium: a brief review. Clin Cardiol 1991; 14(9):708-12.

19. Laky D, Parascan L. Hibernating myocardium, morphological studies on intraoperatory myocardial biopsies and on chronic ischemia experimental model. Rom J Morphol Embryol 2007;48(4):407-13.

20. Page B, Young R, Iyer V, Suzuki G, Lis M, Korotchkina L, et al. Persistent regional downregulation in mitochondrial enzymes and upregulation of stress proteins in swine with chronic hibernating myocardium. Circ Res 2008;102(1):103-12.

21. Wissner E, Mookadam F. Thirty-four years of hibernating myocardium: a case report. J Nucl Cardiol 2007;14(5):745-9.

22. Kupatt C, Hinkel R, von Brühl ML, Pohl T, Horstkotte J, Raake P, et al. Endothelial nitric oxide synthase overexpression provides a functionally relevant angiogenic switch in hibernating pig myocardium. J Am Coll Cardiol 2007;49(14):1575-84.

23. Schneider C, Jaquet K, Malisius R, Geidel S, Bahlmann E, Boczor $\mathrm{S}$, et al. Attenuation of cardiac remodelling by endocardial injection of erythropoietin: ultrasonic strain-rate imaging in a model of hibernating myocardium. Eur Heart J 2007;28(4):499-509.

24. Depre C, Vatner SF. Cardioprotection in stunned and hibernating myocardium. Heart Fail Rev 2007;12(3-4):307-17.

25. Cooper HA, Braunwald E. Clinical importance of stunned and hibernating myocardium. Coron Artery Dis 2001;12(5):387-92.

26. Shah BN, Khattar RS, Senior R. The hibernating myocardium: current concepts, diagnostic dilemmas, and clinical challenges in the post-STICH era. Eur Heart J 2013;34(18):1323-36.

27. Ling LF, Marwick TH, Flores DR, Jaber WA, Brunken RC, Cerqueira MD, et al. Identification of therapeutic benefit from revascularization in patients with left ventricular systolic dysfunction: inducible ischemia versus hibernating myocardium. Circ Cardiovasc Imaging 2013;6(3):363-72.

28. Gaemperli O, Kaufmann PA. PET and PET/CT in cardiovascular disease. Ann N Y Acad Sci 2011;1228:109-136.

29. Brunken RC, Mody FV, Hawkins RA, Nienaber C, Phelps ME, Schelbert HR. Positron emission tomography detects metabolic viability in myocardium with persistent 24-hour single-photon emission computed tomography 201T1 defects. Circulation 1992;86(5):1357-69.

30. Tamaki N, Yonekura Y, Yamashita K, Saji H, Magata Y, Senda M. Positron emission tomography using fluorine-18 deoxyglucose in evaluation of coronary artery bypass grafting. Am J Cardiol 1989;64(14):860-5.

31. Tamaki N, Ohtani H, Yamashita K, Magata Y, Yonekura Y, Nohara R. Metabolic activity in the areas of new fill-in after thallium-201 reinjection: comparison with positron emission tomography using fluorine-18-deoxyglucose. J Nucl Med 1991;32(4):673-8.

32. Chan $\mathrm{KH}, \mathrm{Ng} \mathrm{MKC}$. Is there a role for coronary angiography in the early detection of the vulnerable plaque? Int J Cardiol 2013;164:262-266.

33. Sun $\mathrm{Z}, \mathrm{Xu} \mathrm{L}$. Coronary $\mathrm{CT}$ angiography in the quantitative assessment of coronary plaques. Biomed Res Int 2014;2014:346380.
34. Tillisch J, Brunken R, Marshall, et al. Reversibility of cardiac wall motion abnormalities predicted by positron tomography. $\mathrm{N}$ Engl $\mathrm{J}$ Med 1986;314:884-888.

35. Schinkel AF, Bax JJ, Poldermans D, et al. Hibernating myocardium: diagnosis and patient outcomes. Curr Probl Cardio. 2007;32:375-410.

36. Auerbach MA, Schoder H, Hoh C, Gambhir SS, Yaghoubi S, Sayre JW, Silverman D, Phelps ME, Schelbert HR, Czernin J. Prevalence of myocardial viability as detected by positron emission tomography in patients with ischemic cardiomyopathy. Circulation 1999; 99: 2921-2926.

37. M \& Sun ZH. Diagnostic value of $18 \mathrm{~F}-\mathrm{FDG}$ PET in the assessment of myocardial viability in coronary artery disease: A comparative study with 99mTc SPECT and echocardiography. J Geriatr Cardiol 2014 Sep; 11(3): 229-236.

38. Camici P, Ferrannini E, Opie LH. Myocardial metabolism in ischemic heart disease: basic principles and application to imaging by positron emission tomography. Prog Cardiovasc Dis 1989;32(3):217-38.

39. Wiggers H, Nørrelund H, Nielsen SS, Andersen NH, Nielsen-Kudsk JE, Christiansen JS, et al. Influence of insulin and free fatty acids on contractile function in patients with chronically stunned and hibernating myocardium. Am J Physiol Heart Circ Physiol 2005;289(2):H938-46.

40. Hernandez-Pampaloni M, Bax JJ, Morita K, Dutka DP, Camici PG. Incidence of stunned, hibernating and scarred myocardium in ischaemic cardiomyopathy. Eur J Nucl Med Mol Imaging 2005;32(3):314-21.

41. Gibson RS, Watson DD, Taylor GJ, Crosby IK, Wellons HL, Holt ND. Prospective assessment of regional myocardial perfusion before and after coronary revascularization surgery by quantitative thallium-201 scintigraphy. J Am Coll Cardiol 1983;1(3):804-15.

42. Sun D, Nguyen N, DeGrado TR, Schwaiger M, Brosius FC 3rd. Ischemia induces translocation of the insulin-responsive glucose transporter GLUT4 to the plasma membrane of cardiac myocytes. Circulation. 1994;89(2):793-8.

43. Camici P, Ferrannini E, Opie LH. Myocardial metabolism in ischemic heart disease: basic principles and application to imaging by positron emission tomography. Prog Cardiovasc Dis 1989;32: 217-238.

44. Iozzo P, Chareonthaitawee P, Dutka D, Betteridge DJ, Ferrannini E, Camici PG. Independent association of type 2 diabetes and coronary artery disease with myocardial insulin resistance. Diabetes 2002; 51: 3020-3024.

45. Marinho NV, Keogh BE, Costa DC, Lammerstma AA, Ell PJ, Camici PG. Pathophysiology of chronic left ventricular dysfunction: new insights from the measurement of absolute myocardial blood flow and glucose utilization. Circulation 1996; 93: 737-744.

46. Ferrannini E, Santoro D, Bonadonna R, Natali A, Parodi O, Camici PG. Metabolic and hemodynamic effects of insulin on human hearts. Am J Physiol 1993;264: E308-E315.

47. Chareonthaitawee P, Schaefers K, Baker CR, Turkheimer F, Stegger L, Banner NR, Yacoub M, Bonser RS, Iozzo P, Camici PG, Rimoldi O. Assessment of infarct size by positron emission tomography and [(18)F]2-fluoro-2-deoxy-D-glucose: a new absolute threshold technique. Eur J Nucl Med 2002;29: 203-215.

48. Gerber BL, Ordoubadi FF, Wijns W, Vanoverschelde JL, Knuuti MJ, Janier M, Melon P, Blanksma PK, Bol A, Bax JJ, Melin JA, Camici PG. Positron emission tomography using(18)F-fluorodeoxyglucose and euglycaemic hyperinsulinaemic glucose clamp: optimal criteria for the prediction of recovery of post-ischaemic left ventricular dysfunction: results from the European Community Concerted Action Multicenter Study on Use of(18)F-FluoroDeoxyglucose Positron Emission Tomography for the Detection of Myocardial Viability. Eur Heart J 2001;22: 1691-1701.

49. Camici PG, Prasad SK \& Rimoldi OE. Stunning, Hibernation, and Assessment of Myocardial Viability. Circulation 2008;117:103-114

50. 16. Raja S, Singh B, Rohit MK, et al. Comparison of nitrate augmented Tc-99m tetrofosmin gated SPECT imaging with FDG PET imaging for the assessment of myocardial viability in patients with severe left ventricular dysfunction. J Nucl Cardiol 2012;19:1176-1181. 